\title{
Information Retrieval and Sentimental Analysis with Databricks
}

\author{
Saifuzzafar Jaweed Ahmed \\ Computer Engineering, Dhole Patil College of Engineering Pune, Pune, Maharashtra, India
}

Article Info

Volume 7, Issue 2

Page Number: 459-467

Publication Issue :

March-April-2021

\section{Article History}

Accepted : 20 April 2021

Published : 25 April 2021

\section{ABSTRACT}

With the rapid development of cloud computing, the information increases rapidly. Cheap cloud storage and computing power accelerate the development of massive data, and make the large data information collection and knowledge retrieval become necessary. The percentage of unstructured big data is more than $50 \%$, so it is stored in the form of a file for the most part. Big data is split into many blocks that are stored within the server with some corresponding metadata of storage on the master server. How to collect the big data and keywords and retrieve the information are discussed in this paper.

The proposed methodology is the information retrieval and sentimental analysis mechanism to improve text information retrieval and opinion mining using databricks. Information retrieval and analysis become more popular research fields within the world. Big data is a collection of heterogeneous structured, unstructured and semi-unstructured data. The basic aim of this paper is to present a broad picture of massive Data and to point out how information are often retrieved using evolutionary computation techniques i.e. databricks that help in the information retrieval process in a better way compared to traditional retrieval techniques. This paper also covered the setup and configuration of databricks.

Keywords : Big Data, Information Retrieval, Sentimental analysis, Databricks

\section{INTRODUCTION}

With increased processing power and huge storage space available at an affordable price, the size of scientific data sets has grown to the terabyte and peta byte scale. Efficient and optimised way of storing and retrieving large data volumes is an important goal for the scientific data community. The overall size of the info often necessitates dividing the info across multiple disks o one machine.

Big data refers to datasets whose size is beyond the power of typical management tools to capture, store, manage, and analyze. The traditional database system and data processing tools aren't ready to handle gigantic data at a time. Big data helps to handle this issue.Hence, more effective and sophisticated IR 
techniques for efficient retrieval of information from a large amount of data that will help in decision making is required. These IR techniques should be suitable for user profiles and queries. This simplifies the reclaim and organization of the data for creating knowledge required for the recommender systems. It's preferable if the proficient features of the retrieval model are supportive for decision making and can discover the patterns, trends, and correlations buried in big data.

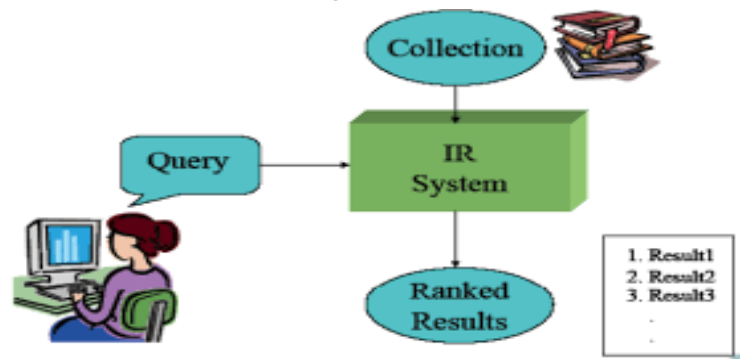

Figure 1. IR System In Big Data

The World Wide Web historically has generated large amounts of data due to increasing demand in internet services and social media platform because of this increase in data generation the techniques for information retrieval plays a really important role. According to Servy, the size of the world-wide-web exceeded 800 million pages in 1999 to 11.5 billion in 2005, and possibly more than 30 billion nowadays. While most data is stored in databases, the current trend is toward big data analytics for storage and retrieval. This technique is driven by mandatory requirements and therefore the potential to enhance the standard of data retrieval and delivery, reducing the time to retrieve the results from these massive quantities of unstructured data (known as 'big data'). Big data in information retrieval is overwhelming not only due to its volume but also due to the range of knowledge types and therefore the speed at which it must be managed. The totality of data related to a user query for information and wellbeing may make up "big data" in the information retrieval systems. It includes all the data which is structured or unstructured data from different data sources like databases, social media posts, including Twitter feeds(so-called tweets), blogs, status updates on Facebook and other platforms. For the large data scientist, there is, amongst this vast amount and array of knowledge, opportunity. Thus, big data analytics applications in information retrieval cash in on the explosion in data to extract insights for creating better-informed decisions, and as a search category are referred to as big data analytics in information retrieval. When big data is analyzed by information retrieval techniques, the above-mentioned associations, patterns and trends are revealed. Healthcare providers and other stakeholders within the information retrieval system can develop a scientific understanding of the knowledge, leading to higher quality information at a lesser time and in better outcomes overall. Many payers are developing and deploying mobile apps that help users to identify the information and save time to retrieve that information, locate providers and improve their efficiency of results retrieved by the system. Via analytics, payers are ready to monitor adherence to usage and reliability trends that cause users benefits.

\section{WHAT IS BIG DATA}

Big data is a term that describes the massive volume of data both structured and unstructured that's growing at an unprecedented rate. The info is existing in Petabytes and is predicted to increase to Exabytes and Yottabytes within the approaching years. The number of data is being exploded with the advancement in technology and there should be proper means to handle such an enormous explosion of data.

The quantity of digital content on the web is found to be around five hundred billion Gigabytes and this number is predicted to double within a year. If we consider the scenario of ten years ago, then one Gigabyte of knowledge seems like a huge amount of data . But with the rise in data and advancement, 
now data is stored in Terabytes or Petabytes. Some even talk about Exabytes or Yottabytes, which may be a trillion Terabytes.

\section{INFORMATION RETRIEVAL}

An information retrieval system is a system that is able to store, retrieve and maintain information. Information has many sorts like text (include numeric and date data), audio, video and other multimedia. Information retrieval modeling is extremely important to assist researchers in designing and implementing an actual efficient data system . Mathematical modeling can be used in several domains such as geographical areas, medical areas.. ..etc. The model of information retrieval helps the user to predict and explain what a user will find relevant given a query.

In daily life when we search something the keywords send to the search engine and submit the search request, that can find matching display web pages step by step, and search engines will analyze the search request detail information. Detailed analysis of the search request is mainly do word processing.

\section{SENTIMENTAL ANALYSIS}

Sentimental analysis may be defined as the classification of a text or document into a positive or a negative class by judging the connotation contained in the text. A positive opinion expressing text is assigned a positive label whereas a negative label denotes a negative opinion. Any objective opinion would be assigned a neutral label.

It is observed that significant work has been done in the domain of product reviews, movie reviews, restaurant reviews blog posts etc. to identify their sentiments but comparatively very less work has been done in the domain of book reviews [7].

Sentimental analysis is the analytical study of people's opinion, their view and emotions.
Sentimental Analysis of tweets from twitter is somewhat different from the normal text processing. Tweets are the 140 character messages and can include various symbols like smiley faces etc.

\section{LITERATURE SURVEY}

Information retrieval (IR) is a technique of finding material (usually documents) of an unstructured nature (usually text) that satisfies an information need from within large collections (usually stored on computers) i.e. big data.

Big Data Retrieval: Taxonomy, Techniques and Feature Analysis[8] by Israr Haneef, Ehsan Ullah Munir, Ghazia Qaiser, Hafiz Gulfam Ahmad Umar elopred the basic of information retrieval and heterogeneous nature of big data also discussed some data analysis methods.

Research On Big Data Information Retrieval Based on Hadoop Architecture (Chen Jie, Chen Dongjie and Huang Bangming): They observed the growth in network and social data across the world and proposed some optimized information retrieval technologies using the Hadoop framework. Also pointed out how clusters may increase the analysis process with different types of clusters.there are also some algorithms like Gzip Algorithm and LZO Algorithm which are used to compress the data.

A survey on information retrieval system modeling using term dependencies and term weighting(Doaa Marouk, Sherinr rady, Nagwa Badr,M.E Khalifa): In this paper there are different information retrieval models which can be used to retrieve the information from big data.

Sentimental Analysis of Book Reviews using Unsupervised Semantic Orientation and Supervised Machine Learning Approaches[7], Vipin Deep Kaur, explored sentimental analysis and also applied 
supervised and unsupervised machine learning on book review.

\section{PROPOSED METHODOLOGY}

In 2020, the accumulated volume of massive data round the world will increase from 4.4 zettabytes to around 44 zettabytes - that's 44 trillion gigabytes and likelihood is that you'll need the proper data tools to seek out the gold beneath.

However, what we ask as big data actually only amounts to 10 percent of the entire data available to organisations; the remaining 90 percent is unstructured, massive and not easy to derive business value out of.This is why big data analytics tools like Apache Spark are essential, as they're designed to figure across massive clusters of databases and servers to explore data during a more efficient way than previously possible.

Azure Databricks fits into the large data equation because of the cloud-optimised version of Apache Spark. It is specifically integrated and optimised for Microsoft Azure, and it had been also designed by the founders of Spark, making it one among the simplest analytics platforms currently available for businesses on the Azure Cloud trying to find an enormous data solution.

\section{A. Databricks}

Databricks is an open source distributed computing framework. Databricks is also founded by the creators of Apache Spark. It's fully managed and with a bunch of goodies on top. Being built by the same people or company who created Spark, it uses the core engine in the best of ways. Most importantly, it removes all the technical steps and parts of getting started.

While Apache Spark is somewhat easy to line up for a technical person, it still requires you to understand network and OS technology. With Databricks, you simply got to click a couple of buttons. Everything else happens under the surface.
This means you'll specialise in your data challenges rather than digging through configuration files. You don't need to care about anything like architecture, setup, or much anything associated with the technical stuff of Apache Spark. Everything is being taken care of for you. That said, knowing what goes on under the hood is useful.

Databricks is additionally cloud optimized and is sweet at scaling both up and down. If you don't run a continuing load 24/7, this is often great. You define the perimeter at start, then Databricks does its magic within the background. That way, you've got power once you need it and no cost once you don't.

This pay-as-you-go model may be a big advantage of Databricks for many companies. You can run your monthly job with an enormous number of nodes then shut them down. Compared to running local servers, the savings are often massive.

You also get an interface that's actually good. It's minimal and easy to use. You and your colleagues can add an equivalent notebook at an equivalent time, as an example. That makes co-development possible even over distance. While working with the built-in notebooks is that the most convenient thanks to interact with Databricks, you'll use other tools also . You just connect using one among many connectors available. You can even use desktop analytics tools like Microsoft Power BI, which works sort of a dream. Since Databricks runs Apache Spark, you'll use it to increase your on-premise setup. This can be good if you've got large, infrequent jobs. It's not an equivalent interface experience, but you would possibly avoid buying more servers for a once-aquarter job.

\section{B. Create an Azure Databricks service}


You would need an Azure subscription to create Databricks like for any other resource on Azure.

You can create new account if you don't have account. After that sign in to Azure portal and click on create a resource and type Databricks in the search box:

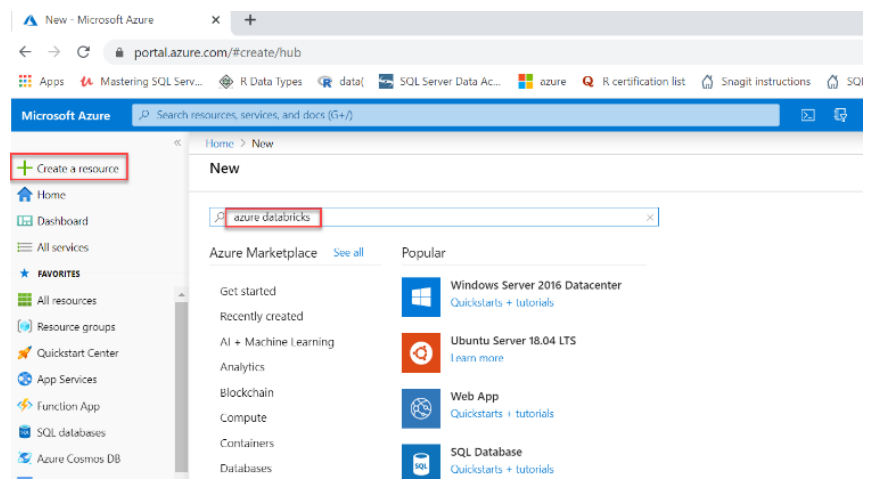

Figure 2. Add resource in databricks dashboard

Click on the Create button, as shown below:

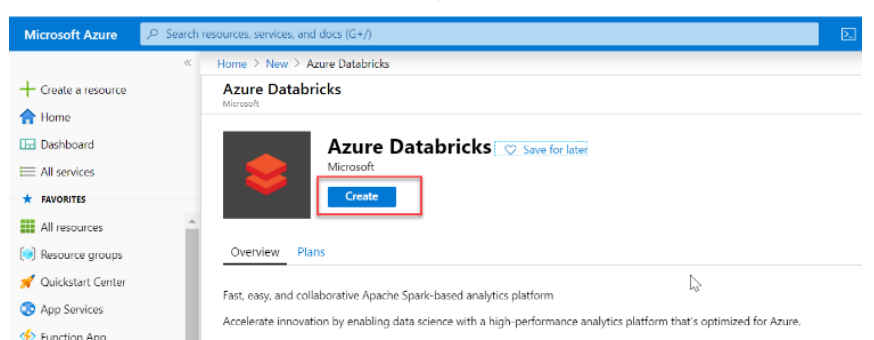

Figure 3. Create resource in databricks dashboard

You will be brought to the following screen. Provide the following information:

- Subscription-Select your subscription

- Resource group - Create the resource new group or used the old one if created before.

- Workspace name - It is the name (azdatabricks) that you want to give for your databricks service, you can give any name.

- Location - Select region where you want to deploy your databricks service, East US or anything else.

- Pricing Tier - Here i am selecting Premium - 14 Days Free DBUs for this demo. To learn about more details on Standard and Premium tiers visit the official website.

Afterward, click on the Review + Create button to review the values submitted and eventually click on the Create button to make this service of databricks:

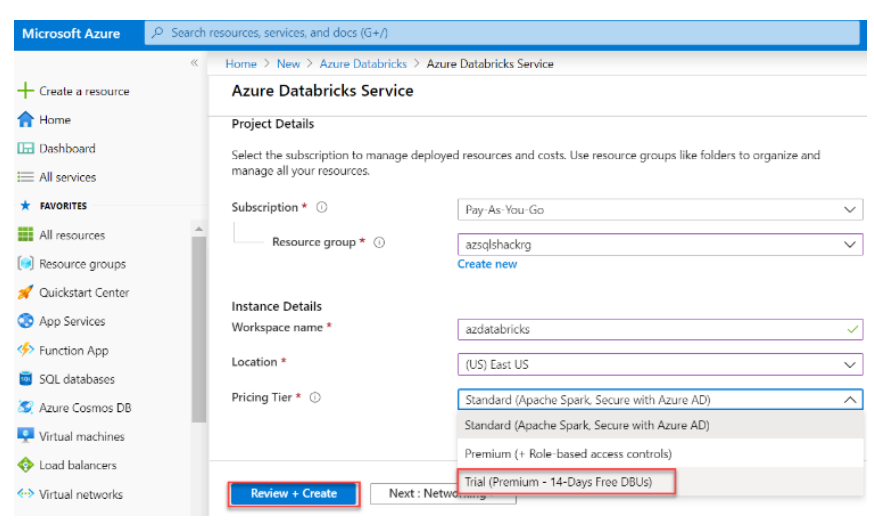

Figure 3. Review and submit service

Once it's created, click on "Go to resource" option within the notification tab to open the service that you simply have just created:

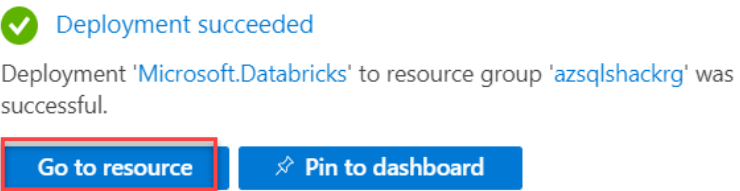

6 minutes ago

You can see several specifics like URL, pricing details and other details etc. about your databricks service on the portal.

Click on Launch Workspace to open the Azure Databricks portal, this is where we will be creating a cluster for further process:

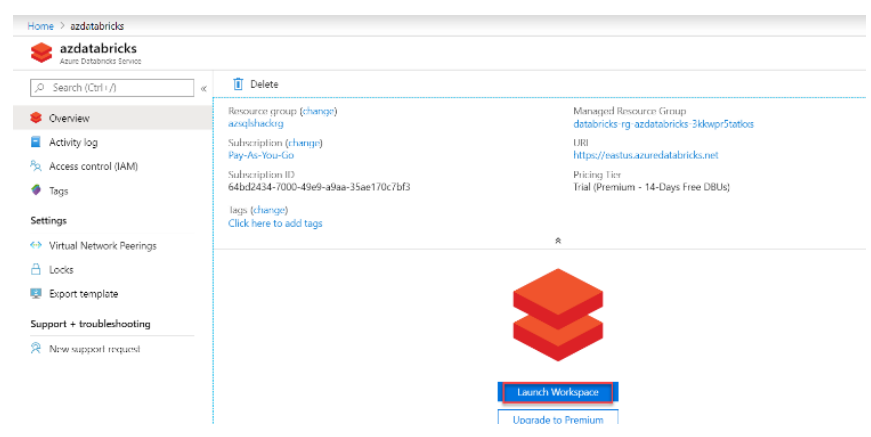

Figure 4. Launch workspace 
You will be asked to sign-in again to launch

Databricks Workspace.

\section{Configuration}

If you think that there are too many steps within the AWS setup, you'll be glad to understand the Azure one is way shorter. Also, Azure Portal is the only one way to deploy it. So you actually won't undergo the Databricks website in the least. Instead, head over to the portal. azure.com.Use your account to log in and make one if you haven't already. Just like with Amazon, there are frequently offers for a few free resources available for newcomers.

Either way, confirm you get to the most portal view.Once you're there, look for Azure Databricks within the search box at the highest. Select it once it comes up and click on the Create Azure Databricks Service button within the center of the screen.

In the setup screen, there are a couple of belongings you got to select. The workspace name is for yourself, the subscription must be connected to a MasterCard, and therefore the location should be close to you physically.

You can create a replacement resource group or use an existing one. Cloud architecture

strategies are outside the scope of this book, but if you're unsure, you ought to use a

new one. The last option is for adding Databricks to at least one of your own virtual networks.

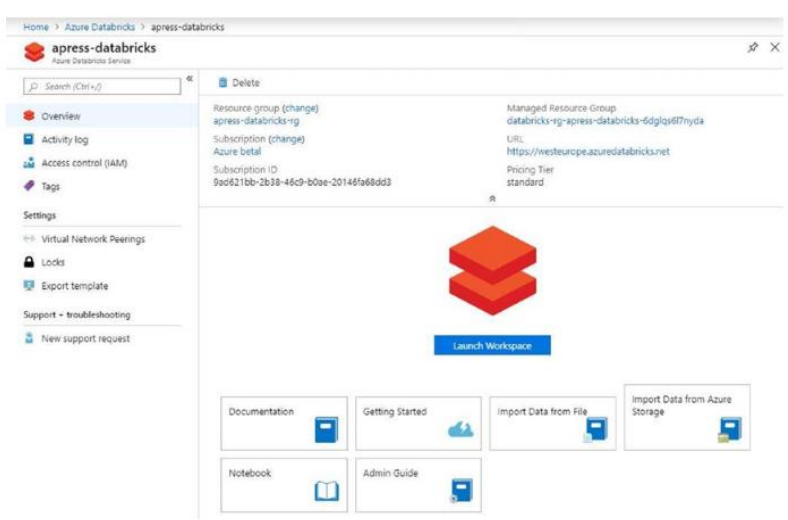

Figure 5. Configuring Databricks on Azure is a breeze Once everything is filled in, just click Create. You'll see the progress bar within the Notifications tab. Wait a few minutes and then click Refresh. Then you'll have the new workspace in the list. Click it to go to the detail page (Above figure). Click LaunchWorkspace to start Databricks.That's it, quite bit easier than doing an equivalent thing with AWS. So if you're totally new to both the cloud and Databricks, i might recommend Azure for this reason. Getting up and running is simply such a lot faster on Microsoft's solution. It should be mentioned that Microsoft actually bought a little stake in Databricks in one of their investment rounds. This could be a reason for his or her apparent specialise in this specific product albeit they need other similar tools in their portfolio.

\section{Clusters: Powering up the engines}

The core of Databricks is in fact the processing power you'll spin up. It's required not only to the underlying Databricks File System but also to run code. Building a cluster is as easy as typing during a name and clicking the Create Cluster button. There are a lot of options, but the default settings are literally ok for many minor use cases and a good place to start.

In the below image (Figure. 6), you see a page where only the Cluster Name field has been changed. Everything else is about to default, which suggests you'll get one among the smallest clusters you can build. It's still quite powerful though, as you'll see

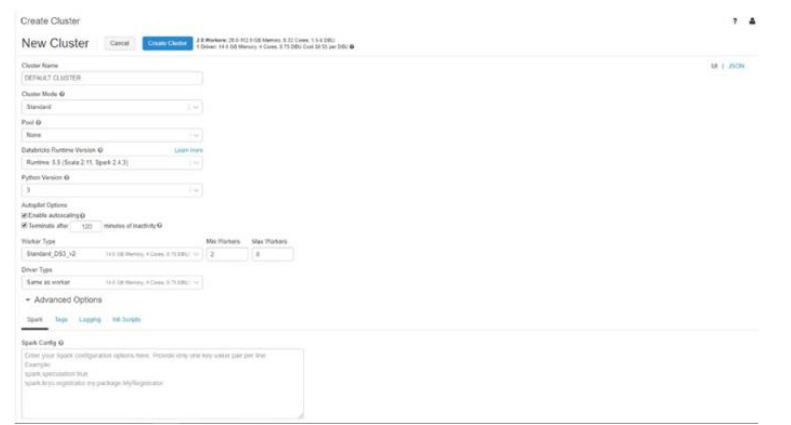

Figure 6. The image is from an Azure setup

Let's check out the various options, from the highest . 
In Cluster Mode, you can decide if this is a Standard or High-Concurrency cluster. You want the HighConcurrency option if you would like to possess an outsized number of users using the cluster at an equivalent time. This is not ideal,but is sometimes necessary. You'll need it for Table Access Control and that is very important. Then you opt if you would like to place the cluster during a pool. This is a fairly new concept for Databricks. The idea is that you simply can keep resources around during a pool to hurry up starting clusters. This is good as starting clusters is otherwise slow, but keep track of costs as this feature is securing machines within the cloud. Databricks, however, doesn't charge anything for the idle instances during a pool.The runtime version is pretty straightforward. You can pick the Databricks/Scala/Spark combination that works for you. Normally you ought to be ready to run with the newest version, but if you would like to make certain the code will work an equivalent over time, it'd be good to select a long-term supported version, or LTS as it's abbreviated within the list. Be aware that older versions in fact lack features newer ones have.

\section{E. Notebooks: Where the work happens}

So the actual or real data processing work, including the data import, is done in notebooks - at least for the most part. As you'll observe later, you can actually connect to Databricks from external tools or any tools and use it as an Apache Spark engine, but notebooks are the main way to talk to the system.

Currently Databricks supports four different languages in the notebooks: Python, R, Scala, and SQL. The first and last of these are the simplest supported ones in terms of security features, while Scala is native. Still, they're all good, so you'll use the one you're most comfortable with unless you would like the extras.

In the start screen there is an option of Create a Blank Notebook. Just for the demo purpose name it Hello World and use SQL as the primary language.
After a few seconds, the work area is created, and you'll be placed in the first textbox of the notebook(Figure. 7)

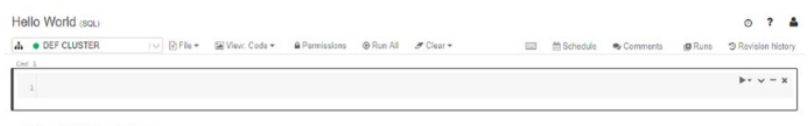

Figure 7. Note that not all keyboard shortcuts work on international keyboards

If you check around, there's a bunch of data and features available. You have the name of the notebook and the main language, at the top. Below it you see the connected cluster (and a choice to change cluster), a couple of menus, then another set of menus.

The main part is the input window of the note book, or the cell as it's called. This is where you enter your code.

input window, or the cell as it's called. This is where you enter your code.

\section{F. Architecture}

So how is Azure Databricks put together in practice? Services of databricks launches and manages worker nodes in each Azure customer's subscription, that's letting customers to management tools within their account at a high level, "Databricks appliance" is deployed as an Azure resource within the customer's subscription when a customer launches a cluster via Databricks. The customer specifies the kinds of VMs to use and the way many, but Databricks manages all other aspects. In addition to the present appliance, a managed resource set is deployed into the customer's subscription that we populate with a VNet, a security group, and a storage account. These are concepts Azure users are familiar with. Once services are ready, users can manage the cluster through the Azure Databricks UI or through features like autoscaling. All metadata is stored in an Azure Database. 


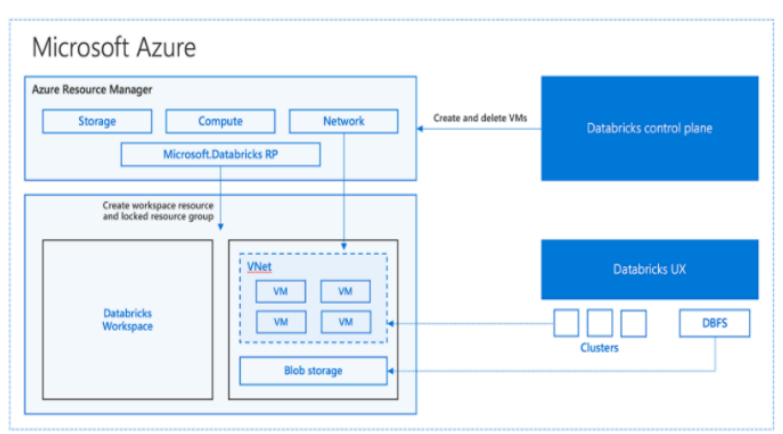

Figure 8. Databricks Architecture

For users, this design means two things. In the first step, they go to simply connect Azure Databricks to any storage resource in their account, e.g. Data Lake. In the second step, Databricks is managed and operate centrally from the Azure center, requiring no additional setup.

\section{RESULT AND DISCUSSIONS}

The Databricks Unified Data Analytics Platform enables Comcast to create rich datasets at a huge scale, optimize machine learning at scale, streamline workflows across teams, foster collaboration, reduce infrastructure complexity, and deliver superior customer experiences.

\section{Visualizations:}

Databricks uses the display and displayHTML functions to support various types of visualizations.

Databricks also supports visualization libraries in Python and $\mathrm{R}$ and also you can install and use thirdparty libraries.

\section{display function:}

Below are the some display function that supports several data and visualization types.

\section{In this section:}

- Data types

- DataFrames

- Images

- Structured Streaming DataFrames

- $\quad$ Plot types
- Choose and configure a chart type

- Chart toolbar

- Color consistency across charts

- Machine learning visualizations

In today's economy, financial services firms are forced to deal with heightened regulatory environments and a spread of market, economic and regulatory uncertainties. Coupled with increasing demand from customers for more personalized experiences and attention on sustainability/ESG, incumbent Banks, Insurers and Asset Managers are reaching the bounds of where their current technology can take them with their Digital Transformation initiatives. It's more critical than ever for institutions to show towards big data and AI to satisfy these demands, and make smarter, faster decisions that reduce risk and protect against fraud. Business leaders and analytics leaders and teams from the Financial Services sector are invited to hitch this industry briefing to seek out new ideas and methods for driving growth.

Recently, Databricks extended its product by adding a new perk which is called as Databricks Delta. Built on top of Apache Spark, Databricks Delta is taken into account as a next-Gen unified analytics engine and is geared towards assisting data engineers to simplify the complex-natured large-scale data management

process.

At present, most of the companies found out their big-data architectures by combining numerous data lakes, data warehouses, and streaming systems, which significantly increases complexities and costs related to system integration and maintenance. The most advanced and new Databricks Delta give you a single data management platform - unified with data lake's scalability, data warehouse's functionality, $\&$ reliability, and low latency live streaming within an integrated system. Apart from this, Databricks Delta also acts as a sensible transactional storage layer which may be 
placeable onto AWS S3 bucket and facilitates processing on an outsized scale across the cloudplatform. As claimed by the parent firm, Delta is an integrated cloud-backed platform that gives outstanding scalability and elasticity by permitting the amalgamation of streaming, data warehousing, execution, and machine learning.

\section{CONCLUSION}

We discussed processes. Setup, configuration of Databricks and its cluster settings. Databricks use to extract meaningful information from the unstructured text through text analysis and Sentimental analysis. It is a very optimized framework to do the data analysis on big data.

\section{REFERENCES}

[1]. Chen Jie, Chen Dongjie "Research On Big Data Information Retrieval Based on Hadoop Architecture", 2014 IEEE Workshop on Electronics, Computer and Applications .

[2]. Doaa Marouk, Sherinr rady, Nagwa Badr,M.E Khalifa," A survey on information retrieval system modeling using term dependencies and term weighting", The 8th IEEE International Conference on Intelligent Computing and Information Systems (ICICIS2017).

[3]. Wang Xiao-shu,Xie Yao,Luo Huan, "Cloud Computing Oriented Retrieval Technology based on Big Data" , 2015 Seventh International Conference on Measuring Technology and Mechatronics Automation

[4]. Peiyun Zhang and Rongjian Xie , "Ontologybased Unstructured Information Organization and Retrieval", World Congress on Software Engineering, DOI 10.1109/WCSE.2009.3059780-7695-3570-8/09 \$25.00 ( 2009 IEEE
[5]. Robert Ilijason, "Beginning Apache Spark Using Azure Databricks", ISBN-13 : 9781484257807

[6]. Christopher D. Manning Stanford University, Prabhakar Raghavan Yahoo! Research, Hinrich Schütze University of Stuttgart, "Introduction To Information Retrieval”, ISBN: 052186571

[7]. Vipin Deep Kaur, "Sentimental Analysis of Book Reviews using Unsupervised Semantic Orientation and Supervised Machine Learning Approaches", Second International Conference on Green Computing and Internet of Things (ICGCIoT)

[8]. Israr Haneef, Ehsan Ullah Munir, Ghazia Qaiser, Hafiz Gulfam Ahmad Umar, "Big Data Retrieval: Taxonomy, Techniques and Feature Analysis",IJCSNS International Journal of Computer Science and Network Security, VOL.18 No.11, November 2018.

\section{Cite this article as :}

Saifuzzafar Jaweed Ahmed, "Information Retrieval and Sentimental Analysis with Databricks", International Journal of Scientific Research in Computer Science, Engineering and Information Technology (IJSRCSEIT), ISSN : 2456-3307, Volume 7 Issue 2, pp. 459-467, March-April 2021. Available at doi : https://doi.org/10.32628/CSEIT2172101 Journal URL : https://ijsrcseit.com/CSEIT2172101 